\title{
Perspektiven protestantischer Rundfunkarbeit in der Dritten Welt
}

\author{
von Frank Kürschner-Pelkmann
}

Die protestantische Rundfunkarbeit in Afrika, Asien und Lateinamerika spiegelt die Vielfalt des Protestantismus wider. Es ist also eine große Zahl von Denominationen an dieser Rundfunkarbeit beteiligt, und es werden extrem unterschiedliche Kommunikationskonzepte verwirklicht. Der Akzent liegt dabei entweder auf weltweiter Verbreitung von Programmen über leistungsstarke regionale Sender oder auf lokalen oder nationalen Sendern oder Programmen, die von den lokalen Kirchen getragen werden. In diesem Beitrag sollen an Beispielen Formen und Perspektiven dieser protestantischen Rundfunkarbeit (vor allem im Bereich des Hörfunks) dargestellt werden, die sich in manchen Bereichen deutlich von katholischer Rundfunkarbeit unterscheidet, in anderen ihr ähnelt und in einer Anzahl von Fällen gemeinsam betrieben wird.

\section{Afrika - Christliche Programme in staatlichen Sendern}

Die meisten afrikanischen Länder haben mit der Unabhängigkeit das Konzept eines staatlichen oder in gewissem Grade vom Staat unabhängigen Rundfunks der britischen und französischen Kolonialherren übernommen. Sehr rasch erwies sich die zentrale Bedeutung des Massenmediums als politisches Machtinstrument, was z. B. darin zum Ausdruck kommt, daß bei Putschversuchen der Rundfunksender neben dem Präsidentenpalast das am meisten umkämpfte Gebäude ist. Aber auch für die tagtäglichen Auseinandersetzungen innerhalb der politischen Führungsschicht und für den Versuch der Regierungen, dem Volk ihre Vorstellungen nahezubringen, ist das Medium von großer Bedeutung. Unter diesen Umständen verwundert es nicht, daß die ineisten nationalen Sender staatsnah sind, und daß private Konkurrenz nicht erwünscht ist. Auf der anderen Seite bieten diese Sender oft qualitativ gute Sendungen zu Entwicklungsthemen wie Gesundheit und verzichten stärker als Privatsender auf Werbespots, die fremde Leitbilder in die Dörfer transportieren und Frustrationen und Landflucht stärken. Je nach Regierung ist in den Rundfunkprogrammen auch Kritik am Bestehenden möglich.

In den meisten Ländern südlich der Sahara bieten die staatlichen Sender den großen Religionsgemeinschaften die Möglichkeit, im Programm zu Wort zu kommen, meist mehrere Stunden pro Woche. Der staatliche Sender stellt die Sendezeit kostenlos zur Verfügung und bezahlt in verschiedenen Ländern auch die Gehälter der Kirchenfunkredakteure. Die Religionsgemeinschaften haben in den meisten dieser Länder in der Programmgestaltung keine Restriktionen zu erwarten, solange sie sich nicht zu politischen Fragen äußern oder andere Religionsgemeinschaften angreifen.

Die christlichen Kirchen haben diese Möglichkeiten von Anfang an sehr intensiv genutzt, während die Muslime erst in den letzten Jahren eine intensive Rundfunkarbeit betreiben, und dies selbstverständlich vor allem in jenen Ländern, in denen es bereits einen signifikanten muslimischen Bevölkerungsanteil gibt. Für die protestantischen

Frank Kürschner-Pelkmann ist Referent in der Kommunikations-Abteilung des Evangelischen Missionswerks in Hamburg. 
Kirchen bestand von Anfang an die Notwendigkeit einer engen Zusammenarbeit, weil die staatlichen Sender nicht bereit waren, mit einer großen Zahl von Kirchen und kirchlichen Gruppen zu verhandeln und eventuell in deren Konflikte verwickelt zu werden. Die nationalen Christenräte haben die Aufgabe übernommen, die Rundfunkarbeit auf protestantischer Seite zu koordinieren und haben auch Kirchen und Gemeinden, die nicht zu den Mitgliedern des Christenrates gehören (in verschiedenen Ländern z. B. Pfingstkirchen) zur Mitwirkung an dieser Rundfunkarbeit eingeladen. In Ländern wie Sambia und Botswana ist es gelungen, zu einer intensiven katholischprotestantischen Zusammenarbeit zu kommen. Die einzelnen Kirchen erhalten einen Anteil an den zu vergebenden morgendlichen Andachtsterminen und Gottesdienstübertragungen, der in etwa dem Anteil der Kirche an der Gesamtzahl der Gläubigen entspricht. Die ökumenische Zusammenarbeit geht in vielen afrikanischen Ländern deutlich über den Grad der Zusammenarbeit in anderen Bereichen hinaus.

Ein Hauptproblem dieser Rundfunkarbeit ist sowohl für Katholiken als auch für Protestanten die Ausbildung von Mitarbeitern, vor allem aber der beteiligten Pastoren und Evangelisten für diese Arbeit. Viele Pastoren kommen das erste Mal in ein Studio, wenn sie eine Andacht sprechen sollen, sind nervös, kennen die spezifischen Bedingungen des Massenmediums nicht etc. In einzelnen Ländern wie Botswana haben die Kirchen eigene kleine Studios eingerichtet, in denen man sich viel Zeit nehmen kann, Aufnahmen zu proben und dann zu wiederholen, bis eine angemessene Sendequalität erreicht ist. Hinzu kommen Kurse für Pastoren und Evangelisten, in denen Grundkenntnisse kirchlicher Hörfunkarbeit vermittelt werden. Unterstützung von außen wird für diese kleinen Studios, für Ausbildungsprogramme sowie für Fahrzeuge und Aufnahmegeräte für Aufnahmen aus der Provinz benötigt. Es handelt sich dabei aber jeweils nur um relativ geringe Beträge. Die christlichen Kirchen und ihre Partner in Übersee sind nicht mit den hohen Kosten für den Betrieb von Sendern oder den Kauf von Sendezeit belastet. Sowohl die einheimischen Kirchen als auch fördernde Stellen in Europa und Nordamerika haben lange gebraucht, um die damit verbundenen Möglichkeiten intensiv zu nutzen und zu fördern.

Bis in die siebziger Jahre hatten die lutherischen Kirchen zusätzlich die Möglichkeit, einen eigenen Sender in Äthiopien zu betreiben,»Radio Voice of the Gospel «. RVOG strahlte Programme aus, die in nationalen Studios (z.B. in Tansania, Madagaskar und Kamerun) aufgenommen worden waren oder die in Addis Abeba selbst produziert wurden. Hierzu gehörten auch Nachrichtensendungen, die wegen ihrer Unabhängigkeit von vielen Afrikanern geschätzt wurden. Nach dem Sturz des äthiopischen Kaisers verstaatlichte die neue Militärregierung den Sender. Nach längeren Überlegungen entschlossen sich die im Lutherischen Weltbund zusammengeschlossenen Kirchen, keinen neuen Sender zu bauen. Stattdessen wurde auf der einen Seite Sendezeit bei bestehenden Sendern in Swasiland, Gabun und auf den Seychellen gekauft, andererseits wurde versucht, die lutherischen Studios in Kommunikationszentren umzugestalten, die neben Hörfunkprogrammen z. B. auch Audiokassetten für Gemeinden und einzelne Christen produzieren. Besonders die lutherische Kirche Madagaskars hat Fortschritte in Richtung auf eine solche umfassende Kommunikationsarbeit erzielt und verbindet Hörfunkprogramme, Kassetten, Poster, Broschüren und andere Medien z.B. mit Maßnahmen innerhalb einer Gesundheitskampagne. Daneben haben die lutherischen Kirchen, wie alle anderen Denominationen, die Möglichkeit, an Programmen für die staatlichen Sender mitzuwirken.

Von den Seychellen aus strahlt die»Far East Broadcasting Corporation « (FEBC) christliche Kurzwellenprogramme nach Afrika aus. Der Sender wird von Christen in 
den USA getragen und ist Teil eines Rundfunkkonzepts, das darauf abzielt, alle Teile der Welt mit christlichen Programmen zu erreichen. Damit sollen alle Menschen die Möglichkeit erhalten, das Wort Gottes zu hören. Ein Problem dieser Hörfunkarbeit besteht darin, daß sie oft an den lokalen Kirchen vorbei betrieben wird. Dies ist einmal durch den großen Sendebereich bedingt, zum anderen aber wohl auch durch das Selbstverständnis nordamerikanischer Rundfunkevangelisten, die ihre Botschaft an alle Enden der Welt bringen möchten. Und so verbreiten diese transnationalen christlichen Sender denn auch nicht nur das Wort Gottes, sondern auch eine Interpretation der biblischen Botschaft durch nordamerikanische Christen oder zumindest unter ihrer Kontrolle.

Ein anderes Problem besteht darin, daß diese Hörfunkarbeit meist nicht verknüpft. ist mit anderen Formen christlicher Kommunikationsarbeit, obwohl sich gerade in den letzten Jahren immer wieder erwiesen hat, wie wirksam christliche Kommunikation dann ist, wenn persönliches Zeugnis, die Nutzung von Kleinmedien und - darauf abgestimmt - der Einsatz von Massenmedien in einem Konzept aufeinander abgestimmt sind.

Außer FEBC betreibtwTrans World Radio e einen christlichen Sender in Afrika, und zwar in Swasiland. Zu erwähnen ist schließlich noch der kleine Missionssender ELWA in Liberia. Die tatsächliche Wirkung dieser Sender ist schwer einzuschätzen. Sie dürfte sehr viel geringer sein als diejenige vergleichbarer Sender in Lateinamerika. Mit wachsender technischer und programmäßiger Qualität der nationalen staatlichen Sender, die, wie erwähnt, auch christliche Programme ausstrahlen, dürfte die Motivation weiter absinken, andere Sender einzustellen (außer BBC für die Nachrichten), zumal wenn diese auf Kurzwelle senden. Diese christlichen Hörfunksender dürften deshalb auch in Zukunft eine eher marginale Rolle in Afrika haben. Um so mehr sind amerikanische Rundfunkevangelisten daran interessiert, in die staatlichen Fernsehprogramme aufgenommen zu werden. Zwar ist die Zahl der Fernsehzuschauer in Afrika noch gering, aber das wird sich mit Sicherheit rasch ändern. Umso wichtiger ist es für die afrikanischen Kirchen und ihre überseeischen Partner, lokale christliche Fernsehproduktionen zu initiieren und zu fördern. Die christliche Botschaft sollte den Menschen in allererster Linie von Christen vermittelt werden, die aus ihrer eigenen Kultur kommen, die ihre Sprache sprechen, ihre Nöte und Sorgen kennen, die Teil ihrer lokalen Kirche sind. Die Übernahme christlicher Fernsehshows aus Kalifornien ist bedenklich, weil die christliche Botschaft so als etwas Fremdes, von außen Kommendes erscheint, verknüpft mit amerikanischem Lebensstil und Wertvorstellungen. Durch die Arbeit der Missionare sind in fast allen Ländern der Welt einheimische Kirchen entstanden, die die Evangelisationsarbeit zu ihrer eigenen Sache gemacht haben (übrigens mit Erfolgen, die die Christen in Europa und Nordamerika bescheiden werden lassen sollten); hinter diese kirchengeschichtliche Stufe sollten wir nicht zurückfallen.

\section{Asien - Vielfalt der Hörfunkarbeit von Minderbeitskirchen}

Die asiatisch-nahöstliche Rundfunksituation zeichnet sich durch eine außerordentliche Vielfalt aus. In Indien gibt es ein großes staatliche Rundfunknetz, auf den Philippinen koexistieren staatliche und kommerzielle Sender, im Libanon sind viele Sender Medieninstrumente politischer Gruppierungen, und in Nordkorea sagt die Regierung, was die Sender zu sagen haben. 
Entsprechend vielfältig ist auch die christliche Rundfunkarbeit. Es gibt eine ganże Reihe von Ländern, in denen keine Programme ausgestrahlt werden können. Dazu gehören die meisten Staaten des Nahen Ostens, aber auch Indien. In der Volksrepublik China werden von Zeit zu Zeit Hörfunk- und Fernsehprogramme mit christlichen Inhalten ausgestrahlt, z.B. zu Weihnachten. Auf der anderen Seite ist die chinesische Bevölkerung die wichtigste Zielgruppe von transnationalen christlichen Hörfunksendern, die von Südkorea, den Philippinen, Taiwan oder Hongkong aus die unerreichten Millionen des Riesenlandes ansprechen wollen. Das hat zu massiven Auseinandersetzungen mit dem Chinesischen Christenrat geführt. Der Christenrat betont, die chinesischen Christen hätten seit 1949 mit Erfolg versucht, ihren Mitbürgern deutlich zu machen, daß das Christentum keine fremde, kolonial-geprägte Religion sei, sondern daß es eine eigenständige chinesische Kirche gebe. Durch Rundfunksendungen von außen würde wiederum der Eindruck erweckt, das Christentum sei etwas Fremdes. Außerdem habe man die Evangelisationsarbeit in die eigenen Hände genommen und zwar erfolgreich, wie das rasche Anwachsen der Gemeinden zeige. Woher nähmen die Christen im Ausland dann aber die Arroganz, per Hörfunk Evangelisation in China betreiben zu wollen, ohne die chinesischen Christen daran zu beteiligen oder zu fragen, ob sie damit einverstanden seien. Außerdem befürchten die im Chinesischen Christenrat zusammengeschlossenen Gemeinden, daß Sendungen von außen den Weg zu der einen protestantischen Kirche behindern können, und zwar dadurch, daß denominationelle Tendenzen gefördert werden und daß Mißtrauen gegenüber dem Christenrat verbreitet wird. Der Lutherische Weltbund hat unter diesen Umständen darauf verzichtet, Hörfunkprogramme nach China auszustrahlen, ebenso verschiedene andere Kirchen und kirchliche Zusammenschlüsse.

Die Philippinen als überwiegend katholisches Land sind ein Zentrum katholischer Hörfunkarbeit in Asien, und Radio Veritas hat wesentlich dazu beigetragen, die Bevölkerung über die tatsächliche Situation des Landes unter Präsident Marcos zu informieren und die Notwendigkeit tiefgreifender Veränderungen bewußtzumachen. Demgegenüber ist die protestantische Hörfunkarbeit auf den Philippinen wesentlich weniger entwickelt und sogar zurückgegangen, nachdem die finanzielle Unterstützung dieser Arbeit aus den US-Kirchen abgenommen hat. Heute gibt es noch zwei protestantische Sender. Der Sender DYSR in der Universitätsstadt Dumaguete hat nur lokale Bedeutung. Mit etwa einem Dutzend bezahlter Mitarbeiter bietet er ein beachtliches Lokalprogramm und könnte sich vielleicht zu einem Modell für christlich getragene, kommunale Hörfunkarbeit entwickeln. Gegenwärtig leidet er allerdings unter einer überalteten technischen Ausstattung und sehr begrenzten Finanzen. Früher wurde der Sender von einem amerikanischen Missionar geleitet. Mit seinem Weggang blieben auch die finanziellen Mittel aus den US-Kirchen aus, leider kein Einzelfall. Heute wird der Sender vom Philippinischen Christenrat getragen. Der andere protestantische Sender»Far Eastern Broadcasting Company « wird weiterhin in enger Zusammenarbeit mit Christen in Nordamerika betrieben und entspechend auch finanziell unterstützt. Er strahlt Programme für den gesamten ostasiatischen Bereich aus.

Ein weiterer FEBC-Sender befindet sich in Seoul in Südkorea. Er wendet sich vor allem an Hörer im Norden der Volksrepublik China. In Südkorea selbst hat er nur einen relativ kleinen Hörerkreis. Hier hat der nationale christliche Sender CBS mit fünf Regionalstationen eine herausragende Bedeutung. Er wird von den protestantischen Kirchen des Landes getragen und gehört zu den wenigen Massenmedien, die nicht von der Regierung kontrolliert werden. CBS darf aber keine Nachrichten aus- 
strahlen und keine Werbespots senden. Nur durch Spenden von Kirchen, Kirchengemeinden und einzelnen Christen in Höhe von mehreren Millionen Mark im Jahr ist es dem Sender möglich, seine Arbeit fortzusetzen.'

In Indonesien und in Sri Lanka erhalten die Kirchen Sendezeit in den staatlichen Rundfunksendern, wobei die Programme selbst produziert werden. In Indonesien gibt es dieses Angebot sowohl auf nationaler als auch auf Provinzebene, und zwar für Hörfunk wie für Fernsehen. Die indonesischen Massenmedien unterliegen einem differenzierten System von Ratschlägen staatlicher Stellen, Selbstzensur und staatlichen Maßnahmen. Das Verbot einer christlichen Tageszeitung im Jahre 1986 zeigt, $\mathrm{daß}$ der Staat nicht zögert, um eine an "Harmonie« orientierte Berichterstattung sicherzustellen. Dies gilt selbstverständlich auch für christliche Hörfunk- und Fernsehbeiträge. In diesem Rahmen steht den Kirchen umfangreiche Sendezeit zur Verfügung. Ähnliches gilt für die christlichen Hörfunkprogramme im benachbarten Sarawak. Angesichts des auch in Südostasien wachsenden Einflusses islamischer Fundamentalisten ist die Möglichkeit christlicher Rundfunkprogramme nicht zu unterschätzen.

In Sri Lanka bemüht sich der Christenrat, in seinen Sendungen einen Beitrag zur Überwindung des Hasses in diesem zerrissenen Land zu leisten. Im Libanon sind christlich geprägte Rundfunksender, wie erwähnt, Teil der politischen und politischreligiösen Auseinandersetzungen.

In den arabischen Ländern besteht vereinzelt für christliche Kommunikationseinrichtungen die Möglichkeit, Rundfunk-Programmangebote zu machen, in denen meist kulturelle und erzieherische Fragen im Mittelpurikt stehen. Im übrigen Asien gibt es für die oft kleinen christlichen Minderheiten zum Teil gar keine und zum Teil geringe Möglichkeiten, christliche Programme über kommerzielle Sender auszustrahlen. Dies ist mit dem Kauf von Sendezeit verbunden, wobei die kleinen Kirchen die Kosten dafür nur mit Unterstützung von außen aufbringen können. Da diese Kosten Jahr für Jahr entstehen, ist eine solche Förderung von außen problematisch, führt sie doch zu einer Dauerabhängigkeit. Auf der anderen Seite wäre es falsch, die relativ wenigen Möglichkeiten für lokale christliche Rundfunkprogramme nicht zu nutzen.

\section{Lateinamerika - Der Einfluß aus dem Norden}

Fast alle Länder Süd- und Zentralamerikas sind katholisch geprägt. Der Protestantismus kam im letzten Jahrhundert in Gestalt von Einwandererkirchen in die Region oder geht auf einzelne protestantische Missionsinitiativen zurück, z.B. auf diejenige der Herrnhuter Brüdergemeinde in Surinam, Nicaragua und in der Karibik.

In den letzten zwei Jahrzehnten ist es zu einer verstärkten Missionsinitiative durch protestantische Kirchen und Gruppen aus den USA gekommen, von denen sich viele der Massenmedien Hörfunk und Fernsehen bedienen. In Ländern wie Guatemala haben diese meist fundamentalistischen Gruppen erheblichen Einfluß gewonnen. In einem weitgehend christianisierten Teil der Welt können diese Gruppen kaum»Pioniermission « im üblichen Sinne betreiben, sondern die katholische und die schon länger bestehenden protestantischen Kirchen müssen zurecht befürchten, daß ihre Gemeindemitglieder abgeworben werden. Sie haben dabei die Erfahrungen in den USA vor Augen, wo die»electronic church « Anhänger unter den Gläubigen der bestehenden katholischen und der protestantischen Kirchen sucht und findet. Selbst wo es 
nicht darum geht, Gläubige um sich zu scharen, müssen die Betreiber dieser Hörfunkund Fernsehevangelisten versuchen, Geld von den Zuhörern und Zuschauern zu erhalten. Geld, das für die Arbeit der Kirchen dann fehlt. Aber in der harten Konkurrenz der Rundfunkevangelisten der »electronic church« kann nur überleben, wer die hohen Kosten für Studio, Sendezeit, Verwaltung, Werbung etc. durch Spenden abdecken kann. Da aber die Hörfunk- und Fernsehevangelisten den gleichen begrenzten Zuhörer- und Zuschauerkreis ansprechen und nicht selten eine "electronic church"Sendung auf die andere folgt, ist der Kampf um die Spenden hart, fast müßte man sagen gnadenlos.

Der lateinamerikanische und karibische Spenden"markt" ist für diese Evangelisten zur Zeit noch nicht sonderlich lohnend, aber die Kosten für die Ausstrahlung der Programme sind niedrig, und die Zukunftsaussichten werden günstig eingeschätzt.

Welche Auswirkungen hat diese Medien «invasion « in Lateinamerika und der Karibik? Zunächst einmal ist sie Teil der kulturellen und sozialen Überfremdung der Region durch den mächtigen Nachbarn im Norden, was um so deutlicher wird, als manche Evangelisten ihre Rundreisen und Auftritte in Fußballstadien nach außen hin sichtbar von multinationalen Unternehmen aus den USA »sponsorn « lassen. Die Befürchtungen vieler Lateinamerikaner vor und ihre Vorbehalte gegenüber dem übermächtigen Einfluß aus dem Norden richten sich somit auch gegen diese christlichen Initiativen und Gruppen.

In den einzelnen Ländern bedeutet das Vordringen der melectronic church« eine Belastung für das evangelisch-katholische Verhältnis. Nicht wenige Katholiken identifizieren den Protestantismus nunmehr mit den westlich-individualistischen, fremden Botschaften der melectronic church», und auch Priester und Bischöfe können nicht umhin, mit Sorge zu sehen, wie protestantische Gruppen sich mehr oder minder offen bemühen, Katholiken für sich zu gewinnen. In Ländern wie Brasilien, wo große Teile der katholischen Kirche arm sind oder sich mit den Armen identifizieren, kann zudem nur mit Erstaunen und vielleicht auch Zorn zur Kenntnis genommen werden, wie amerikanische Fernsehprediger aus der guten Nachricht für alle Menschen, besonders aber für die Armen, eine Botschaft einer selbstzufriedenen nordamerikanischen Mittel- und Oberschicht machen, für die der status quo heilig und Befreiung eine kommunistische Bedrohung ist.

Viele protestantische Kirchen in Lateinamerika mögen sich weder mit der Expansion der »electronic church" noch mit ihrer Botschaft abfinden. Kirchen wie die lutherische Kirche Brasiliens haben in den letzten Jahren sehr viel stärker ihre Verantwortung in der Gesellschaft erkannt und wahrgenommen. Sehr häufig hat dies zu einer engen Zusammenarbeit von katholischen und protestantischen Christen geführt in Sozialprogrammen, im Rechtsbeistand für Menschenrechtsopfer, in genossenschaftlichen Programmen und bei Kommunikationsprogrammen.

Das Gewicht der katholischen Kirche in Lateinamerika und der Karibik findet seinen Niederschlag in einer großen Zahl von Kommunikationsprogrammen, einschließlich Rundfunksendern. Demgegenüber ist die Zahl der von einheimischen protestantischen Kirchen getragenen Hörfunksender klein. Der lateinamerikanische Protestantismus ist in zahlreiche Denominationen gespalten, die erst allmählich zu gemeinsamen Kommunikationsprogrammen finden. 
Ein gelungenes Beispiel für solche Bemühungen ist die Kommunikationsabteilung der theologischen Hochschule ISEDET in Buenos Aires. Diese Hochschule wird von den großen protestantischen Kirchen Argentiniens getragen und bietet so gute Voraussetzungen für eine gemeinsame Kommunikationsarbeit. In den sechziger Jahren wurden Programme für einen Missionssender in der Karibik produziert, der die Programme tber ganz Zentral- und Südamerika verbreitete. Die Wirkung war nicht meßbar, die Verantwortlichen in Buenos Aires waren eher skeptisch. Außerdem gab es Konflikte mit den Verantwortlichen des Senders, denen die Programme aus Buenos Aires zu okumensich waren. Mit Unterstützung des Evangelischen Missionswerkes (EMW) in Hamburg wurde die Rundfunkarbeit von ISEDET neu strukturiert. Man verzichtete auf die kontinentale Ausstrahlung der Programme. Stattdessen werden jetzt tägliche Programme für private Sender in Argentinien und einigen Nachbarländern produziert. ISEDET finanziert mit Unterstützung des EMW die Produktion der Programme. Lokale Kirchengemeinden tragen die Ausstrahlungskosten für die Programme in ihrer Stadt oder ihrem Landkreis. Sie ergänzen die von ISEDET bereitgestellten Andachten um lokale kirchliche Nachrichten und Ankündigungen. Die Pastoren der beteiligten Gemeinden werden in die Herstellung der Programme einbezogen, wobei allerdings, wie auf vielen anderen Gebieten in Argentinien, auch ein Übergewicht auf Buenos Aires liegt. Neben den Hörfunkprogrammen werden inzwischen auch Kommunikationsausbildungskurse angeboten, es werden AV-Medien produziert, Medien verliehen und erste Video- und Fernsehprojekte verwirklicht. Gleichzeitig ist die Kommunikationsausbildung in das Theologen-Ausbildungsprogramm der Hochchule integriert worden, so daß die jungen Theologen jetzt mehr über die Wirkung von Massenmedien erfahren und zugleich lernen, selbst Medien herzustellen sowie an kirchlichen Hörfunk-und Fernsehprogrammen mitzuwirken. So soll die Verknüpfung von Gemeindearbeit und kirchlicher Medienarbeit noch intensiver werden.

Die lutherische Kirche Brasiliens betreibt mehrere eigene Rundfunksender, mit Problemen, die sicher auch den katholischen Sendern nicht unbekannt sind. Die wirtchaftliche Depression in Lateinamerika mit hohen Inflationsraten, sinkender KaufIraft der Bevölkerung, wachsenden Sozialproblemen etc. hat bei den meisten privaten Betreibern zu Problemen geführt, wobei kirchliche Sender besonders betroffen wurden. Kommerzielle Sender legen sich keine Restriktionen bei der Werbung für Zigaretten oder Alkohol auf, sie vermischen meist skrupellos Nachrichten und PR-Texte ton Unternehmen, sie kommen mit einer kleinen Mitarbeiterzahl aus, weil ihr redaktioneller Anspruch oft minimal ist, und sie versuchen, möglichst kaufkräftige Hörertreise anzusprechen, weil dies von der werbenden Wirtschaft honoriert wird. Kirchlithe Sender dagegen sind bei der Aufnahme von Werbung wählerisch, vermischen selten werbende Texte und Nachrichten, berichten dafür sehr viel häufiger als kommerzialisierte Konkurrenten über Mißstände im Wirtschaftsleben und erreichen vor allem irmere, und d.h. weniger kaufkräftige Bevölkerungskreise. In Zeiten, in denen selbst die durch und durch kommerziellen Sender finanzielle Probleme habe, kann es den tirchlichen, ebenfalls auf Werbeeinnahmen angewiesenen Sendern nicht gut gehen, and so gerieten die lutherischen Sender in Brasilien Anfang der achtziger Jahre in eine solche Finanzkrise, daß ihr Weiterbestehen stark gefährdet war. Nur große lokale und internationale kirchliche Anstrengungen machten es möglich, die Arbeit fortzuführen. Für diese wie für andere kirchliche Sender fällt positiv ins Gewicht, daß ihr qualitativ gutes Programm und ihr Engagement für die ärmere Bevölkerung ihnen große Hörerkreise sichern, die dann trotz begrenzter Kaufkraft interessant für die werbende Wirtschaft sind. 
Neben dem wirtschaftlichen Überleben ist es für diese kirchlichen Sender selbstverständlich wichtig, neue Formen der Programmarbeit zu entwickeln, die es ermöglichen, die befreiende Botschaft des Evangeliums für die Hörer lebendig werden zu lassen. Dazu gehört, in den Andachten auf die konkreten Erfahrungen und Probleme der Hörer einzugehen, ihre kulturelle und soziale Situation ernstzunehmen und sie auch selbst zu Wort kommen zu lassen. Solche lokalen christlichen Rundfunksender und -programme sind die vielleicht wirksamste Alternative zu den kontinentalen und globalen Programmangeboten der "electronic church «. Dabei bleibt zu hoffen, daß es bei solchen lokalen Initiativen noch stärker als bisher zu einer katholisch-evangelischen Zusammenarbeit kommt.

\section{Perspektiven für die nächsten Jabre}

Dieser Überblick konnte bei weitem nicht alle Formen protestantischer Rundfunkarbeit darstellen, es sind aber wohl trotzdem einige Tendenzen erkennbar geworden, aus denen sich konzeptionelle Konsequenzen für die Rundfunkarbeit der protestantischen Kirchen in der Dritten Welt und ihre Zusammenarbeit mit katholischen Kirchen und Gemeinden ergeben.

1. Christliche Rundfunkarbeit ist dann besonders wirksam, wenn sie mit anderen Formen christlicher Kommunikationsarbeit verknüpft wird. Deshalb sollte sie, wo immer möglich, in der lokalen kirchlichen Arbeit verankert sein.

2. Für die Vermittlung und Auslegung der biblischen Botschaft ist es erforderlich, die lokale Situation zu kennen und möglichst auch in ihr zu leben. So kann bei der Auslegung der Bibel, in Andachten und Meditationen auf die Erfahrungen, Probleme und Hoffnungen der Menschen eingegangen werden. Dies ist in weltweit verbreiteten Hörfunkprogrammen kaum möglich.

3. Die sehr unterschiedlichen Rundfunkstrukturen und politischen und kirchenpolitischen Verhältnisse in den einzelnen Ländern machen es erforderlich, jeweils genau zu prüfen, welche Form christlicher Rundfunkarbeit am sinnvollsten ist. Wo in staatlichen und öffentlich-rechtlichen Sendern kostenlos Sendezeit zur Verfügung gestellt wird, sollte dieses Angebot optimal genutzt werden, wozu unter Umständen Investitionen in Ausbildungsprogramme, kleine kirchliche Studios, tragbare Aufnahmegeräte u.ä. erforderlich sind. Wo diese Möglichkeit nicht besteht, sind Möglichkeiten des Erwerbs oder Aufbaus eines eigenen kirchlichen Senders oder der Kauf von Sendezeit zu prüfen. Der Kauf von Sendezeit bedeutet, auch langfristig relativ hohe laufende Kosten zu haben. Andererseits muß sehr realistisch und kritisch geprüft werden, welche finanziellen Perspektiven ein eigener Sender hat, der sich primär an den Bedürfnissen der armen Bevölkerung orientiert. Die Ergebnisse werden von Land zu Land unterschiedlich sein, wobei auch zu berücksichtigen ist, welche Belastung ein eigener Sender für kleinere Kirchen sein kann.

4. In Ländern, in denen die Kirchen keine Möglichkeit haben, christliche Hörfunkprogramme zu senden, ist im Einvernehmen mit den lokalen Kirchen die Möglichkeit zu prüfen, Programme über einen Sender im Ausland auszustrahlen. Dabei ist aber zu berücksichtigen, wie stark die meist über Kurzwelle ausgestrahlten Programme wirklich Hörer finden, wie das Verhältnis von finanziellem Aufwand und Ergebnis ist, in welchem Programmumfeld die eigenen Programme stehen, und ob es lokal andere und bessere Formen christlicher Kommunikationsarbeit gibt als die Ausstrahlung von Hörfunkprogrammen aus dem Ausland. 
5. Wo immer möglich, sollte angestrebt werden, eine möglichst breite denominationelle Trägerschaft für christliche Hörfunkprogramme zu finden und dabei auch eine evangelisch-katholische Zusammenarbeit zu suchen. Das gilt insbesondere auch in den Ländern, in denen fundamentalistische Rundfunkevangelisten die ökumenische Gemeinschaft dadurch belasten, daß sie Christen unterschiedlicher Konfession für sich zu gewinnen versuchen.

6. Die verstärkte Verbreitung von Fernsehgeräten sowie die globale Verbreitung auch religiöser Programme stellt die Kirchen in der Dritten Welt und ihre ökumenischen Partner vor neue Herausforderungen. Die lokale Produktion christlicher Fernsehprogramme ist teuer und schwierig, in ökumenischer Zusammenarbeit aber möglich. Als Ergänzung von Gemeindearbeit und von anderen Formen christlicher Kommunikationsarbeit ist die Präsenz im Fernsehen wichtig.

7. Christliche Kommunikationsarbeit in Hörfunk und Fernsehen kann sich auch in der Dritten Welt nicht auf die Gestaltung kirchlicher Programme beschränken. Die Kirchen und die einzelnen Christen können sich bemühen, an einer Medienstruktur mitzuwirken, in der Hörfunk und Fernsehen mehr sind als ein Propagandainstrument der Regierung oder mächtiger Wirtschaftskreise. Ein solches christliches Engagement für Ehrlichkeit, Redlichkeit, für die Mitwirkung der SprachlosGemachten an den Massenmedien führt zu Konflikten, aber es gehört zum christlichen Zeugnis in der heutigen Welt.

\section{Anmerkungen}

1 Vgl. Frank Kürschner-Pelkmann: CBS - ein christlicher Rundfunksender in Korea. In: CS 18:1985, 260-270.

\section{Summary}

The Protestant radio- and television work in Africa, Asia and Latin America reflects the diversity of Protestantism. In many fields, this work is different from Catholic initiatives of the same kind, and in a number of cases there is fruitful cooperation. Both churches, for example, have common education centres in some African countries and produce common programmes which are transmitted via national broadcasting stations. The preferred form of Christian communication on this continent is to make use of national offers leaving out of consideration some broadcasting stations of fundamentalists. In Asia, however, the Protestant radio work shows a diversity of initiatives, even by broadcasting stations of its own. In Latin America, the two major Christian churches are challenged by the influence of North American sects. The melectronic church « from the U.S.A. is trying to lure believers away primarily from the Catholic church by means of capitalistic methods. Just in this continent, the Catholic church itself has many broadcasting stations of its own. Under seven points, the author finally summarizes the perspectives of Protestant radio-and television work in the Third World for the future.

\section{Résumé}

Le travail de radiodiffusion et de télévision de l'Eglise protestante en Afrique, Asie et en Amerique latine reflècte la diversité du Protestantisme. Il se différencie dans plusieurs domaines d'initiatives catholiques de même genre et dans un certain nombre de cas enregistre-t-on une fructueuse coopération. Ainsi dirigent les deux Eglises dans certains pays africains des centres de formation et produisent des programmes communs qui sont emis par des stations d'émission 
étatiques. L'utilisation des possibilités offertes par l'Etat est le moyen privilegié de cornmunication chrétienne dans ce continent, à l'exception de certaines stations-radio des Fondamentalistes. En Asie, par contre, se caracterise le travail de radiodiffusion de Protestantes par la diversité des initiatives, mêmes à travers certains centres émetteurs. Les deux grandes Eglises chrétiennes en Amérique latine sont menacées par l'influence de sectes nordamericains. L'welectronic church* des USA essaie avec des méthodes capitalistes de détourner surtout les croyants de l'Eglise catholique, qui a elle-même ses propres centres de radiodiffusion exactement dans ce continent. L'auteur explique enfin en sept points les perspectives de travail de radiodiffusion et de télévision des protestants d'avenir dans le tiers monde.

\section{Resumen}

El trabajo de la Radio y Televisión protestante en Africa, Asia y Latinoamérica refleja la diversidad del protestantismo. En muchos sectores se distingue de las iniciativas católicas semejantes y en algunos casos existe una cooperación fructuosa. En algunos paises de Africa, por ejemplo, las dos Iglesias tienen centros comunes de formcaión y producen programas comunes, que son transmitidos por las emisoras nacionales. Aparte de algunas emisoras de Radio fundamentalistas, el camino preferido de comunicación cristiana en este continente consiste en la utilización de las ofertas nacionales. Al contrario, en Asia, el trabajo de la Radio protestante muestra una diversidad de iniciativas, también a través de emisoras propias. La influencia de sectas norteamericanas provoca a las dos mayores Iglesias cristianas de latinoamérica. La welectronic church * de los EEUU intenta a través de métodos capitalistas, atraer fieles, sobre todo de la Iglesia católica, la cual, precisamente en este continente, tiene un número bastante grande de emisoras de Radio propias. En siete puntos encierra el autor finalmente las perspectivas del trabajo de la Radio y la televisión protestante en el tercer Mundo para el futuro. 